\title{
Discordance between antral follicle counts and anti-Müllerian hormone levels in women undergoing in vitro fertilization
}

Yangyang Zhang, Yang Xu*, Qing Xue, Jing Shang, Xiuli Yang, Xuemin Shan, Yanrong Kuai, Sheng Wang and Cheng Zeng

\begin{abstract}
Background: In general, anti-Müllerian hormone (AMH) is positively associated with antral follicle count (AFC). However, there is often discordance between the AMH level and AFC in clinical practice. In cases of discordance, which indicator should be chosen to predict ovarian response and subsequently develop an ovulation induction protocol? The objective of this study was to investigate which indicator was more accurate in predicting ovarian response and pregnancy outcomes when the AMH level and AFC were discordant.

Methods: A total of 1121 infertile women undergoing IVF/ICSI were recruited in this study. During the study period, patients were subjected to individualized controlled ovarian hyperstimulation $(\mathrm{COH})$ protocols according to specific characteristics. The AMH levels and AFCs were measured on days 2-3 of the menstrual cycle. Serum samples were obtained to determine AMH levels. Transvaginal ultrasound was performed to determine the AFC. All patients were divided into four groups: Group A had AFCs and AMH levels in the normal range; Group B had normal AFCs and low AMH levels; Group C had low AFCs and normal AMH levels; and Group D had low AFCs and AMH levels.

Results: Two hundred three women (18.11\%) showed discordant AFCs and AMH levels. In the two groups with discordant AFCs and AMH levels, namely, Group B and Group C, the oocyte yield, good-quality embryo rate and clinical pregnancy rate were significantly higher in Group B than in Group C. The incidence of poor ovarian response (POR) was significantly lower in Group B than in Group C. According to the stratified analysis of age, for the three categories above the age of 30, oocyte yield was higher in Group B than in Group C. In all age categories, the clinical pregnancy rate was higher in Group B than in Group C.

Conclusions: Our study demonstrated that approximately one in five patients in clinical practice showed discordance between AFCs and AMH levels. In view of the AFC being better than AMH for predicting POR, the AFC should be the preferred indicator for predicting ovarian response to subsequently develop an optimal individualized $\mathrm{COH}$ protocol.
\end{abstract}

Keywords: Antral follicle count, Anti-Müllerian hormone, Ovarian response, Clinical pregnancy rate

\footnotetext{
* Correspondence: xuyangm@126.com

Department of Obstetrics \& Gynecology, Peking University First Hospital, Beijing 100034, China
}

(c) The Author(s). 2019 Open Access This article is distributed under the terms of the Creative Commons Attribution 4.0 International License (http://creativecommons.org/licenses/by/4.0/), which permits unrestricted use, distribution, and reproduction in any medium, provided you give appropriate credit to the original author(s) and the source, provide a link to the Creative Commons license, and indicate if changes were made. The Creative Commons Public Domain Dedication waiver (http://creativecommons.org/publicdomain/zero/1.0/) applies to the data made available in this article, unless otherwise stated. 


\section{Background}

In the process of controlled ovarian hyperstimulation $(\mathrm{COH})$, the key steps are to evaluate ovarian reserve function, predict ovarian response, and develop an optimal individualized $\mathrm{COH}$ protocol. Only in this way, it is possible to obtain an appropriate quantity of oocytes and attain high-quality oocytes, and ultimately produce a sufficient number of high-quality embryos and improve the clinical pregnancy rate. There are many indicators for predicting ovarian response and pregnancy outcomes, including age, hormone levels (basal follicle stimulating hormone (FSH), oestrogen $\left(E_{2}\right)$, antiMüllerian hormone (AMH), etc.) and ultrasound indicators (ovarian volume, antral follicle count(AFC), etc.) [1]. Current studies have shown that the AMH level and AFC have higher predictive value for poor ovarian response (POR) than other indicators [2, 3], and the accuracy of prediction is consistent [4].AMH is produced by the granulosa cells of pre-antral and small antral follicles, and its level is not affected by the menstrual cycle [5] or exogenous hormonal supplementation [6].Therefore, $\mathrm{AMH}$ levels can better represent the number of primordial follicles and reflect ovarian reserve function. The AFC refers to the number of follicles with diameters of $2 \mathrm{~mm}$ to $9 \mathrm{~mm}$ [7]; these follicles begin to develop after recruitment in the luteal phase of the previous cycle and generally reflect the number of follicles that will continue to mature during the ovulation treatment cycle. In general, AMH is positively associated with AFC; that is, patients with good ovarian reserve function have high AMH and AFC values, and those with poor ovarian reserve function have low values. However, there is often discordance between the AMH level and AFC in clinical practice. In cases of discordance, which indicator should be chosen to predict ovarian response and subsequently develop an ovulation induction protocol? The objective of this study was to investigate which indicator was more accurate in predicting ovarian response and pregnancy outcomes when the AMH level and AFC were discordant.

\section{Methods \\ Patients}

A total of 1121 infertile women undergoing in vitro fertilization (IVF) /intracytoplasmic sperm injection (ICSI) were recruited in this study from January 2016 to December 2017 at the Reproductive and Genetic Medical Center of Peking University First Hospital. Women with a history of ovarian surgery, polycystic ovarian syndrome (PCOS), hormonal therapy in the past 6 months or other endocrine diseases, including diabetes mellitus, thyroid disease, and hyperprolactinemia were excluded from this study. This study was approved by the Clinical
Research Institutional Review Board of Peking University First Hospital, and all patients provided informed consent.

\section{$\mathrm{COH}$ protocols}

During the study period, each patient was subjected to an individualized $\mathrm{COH}$ protocol according to specific characteristics, such as ovarian reserve and follicle size. Gonadotropin therapy was performed, and follicles were regularly monitored by transvaginal ultrasound. Recombinant human chorionic gonadotropin (HCG) was administered subcutaneously when the leading follicle was $18-20 \mathrm{~mm}$ in diameter. Oocytes were retrieved by transvaginal ultrasound-guided follicular aspiration within approximately $36 \mathrm{~h}$ after HCG administration. Oocytes were fertilized by conventional IVF/ICSI, and embryos were transferred under abdominal ultrasound guidance on day 3 after oocyte retrieval. HCG tests were performed on day 14 after ET, and if the result was positive, luteal support was continued as before until 10 weeks of gestation. Clinical pregnancy was defined as the presence of an intrauterine gestational sac 4 weeks after ET. The transplantation was cancelled for the following reasons: (1)to prevent the occurrence of ovarian hyperstimulation syndrome (OHSS); (2) when no transplantable embryos were obtained; (3) to accumulate embryos; or (4) when progesterone levels were $>2.5 \mathrm{ng} / \mathrm{ml}$ on HCG day.

Patients were divided into four groups according to the boundaries for the AFC and AMH level in the ovarian reserve test provided by the "Bologna criteria" [8]:

Group A, AFC $\geq 7$ and $A M H \geq 1.1 \mathrm{ng} / \mathrm{ml}, 611$ women (both AFCs and AMH levels in the normal range); Group B, AFC $\geq 7$ and $\mathrm{AMH}<1.1 \mathrm{ng} / \mathrm{ml}, 85$ women (normal AFCs and low AMH levels); Group C, AFC $<7$ and $\mathrm{AMH} \geq 1.1 \mathrm{ng} / \mathrm{ml}, 118$ women (low AFCs and normal AMH levels); Group D, AFC $<7$ and $\mathrm{AMH}<1.1 \mathrm{ng} / \mathrm{ml}, 307$ women (low AFCs and low AMH levels).

The following criteria were used to define ovarian response according to oocyte yield [9]: POR, oocyte yield< 4; normal ovarian response, oocyte yield $\geq 4$ and $\leq 15$; and high ovarian response, oocyte yield $>15$.

\section{Assessment of AMH levels and AFCs}

Serum samples were obtained on days $2-3$ of the spontaneous menstrual cycle prior to one month of IVF/ICSI treatment in agonist protocol cycles for serum AMH measurements. In antagonist cycles, serum samples were obtained on days $2-3$ of the treatment cycle. Serum samples were separated within $1 \mathrm{~h}$ after collection and stored at $-80{ }^{\circ} \mathrm{C}$ until analysis for AMH. Serum AMH 
levels were analysed by enzyme-linked immunosorbent assay (ELISA).

Transvaginal ultrasound was performed to assess the AFC on days 2-3 of the treatment cycle using an Aloka SSD-1000 (Japan) with a $5 \mathrm{MHz}$ transvaginal probe by one of four doctors for each case. Operators underwent uniform training prior to the start of the study to reduce errors caused by different operators. Follicles measuring $2 \mathrm{~mm}-10 \mathrm{~mm}$ in diameter were counted in both ovaries to determine the AFC. The total number of follicles in both ovaries was used as the AFC.

\section{Statistical analysis}

All analyses were performed with the Software Package for Social Sciences (SPSS) version 13.0 for Windows. All normal distribution measurement data are expressed as the mean \pm standard deviation (SD). Comparisons between two groups were analysed with independent sample t-tests, comparisons among multiple samples were analysed by variance analysis, and intergroup multiple comparisons were analysed with the Bonferroni correction. The count data were analysed by chi-square test. $P<0.05$ was considered statistically significant.

\section{Results}

In this study, the AFCs and AMH levels of 918 women $(81.89 \%)$ were concordant in that they were both either normal or low. The AFCs and AMH levels of 203 women $(18.11 \%)$ were discordant. Eighty-five women (7.58\%) had normal AFC values and low AMH values
(Group B), and 118 women (10.53\%) had low AFC values and normal AMH values (Group C). Table 1 summarizes the characteristics of the groups.

As shown in Table 1, for all study subjects, patient age $(p<0.001)$, FSH level $(\mathrm{p}<0.001)$ and POR rate $(\mathrm{p}<$ $0.001)$ progressively increased, and oocyte yield $(\mathrm{p}<$ 0.001) progressively decreased from Group A to Group B to Group C and to Group D. The clinical pregnancy rates of Group A and Group B were significantly higher than those of Group C and Group D.

In the two groups with discordant AFCs and $\mathrm{AMH}$ levels, namely, Group B (normal AFCs and low AMH levels) and Group C (low AFC and normal AMH levels), the oocyte yield, good-quality embryo rate and clinical pregnancy rate were significantly higher in Group B than in Group C. The incidence of POR in Group B was significantly lower than that in Group C.

Patients were stratified into the following age categories: $\leq 30.0,30.1-37.9,38.0-41.9$, and $\geq 42.09$ years. As shown in Table 2, the incidences of AMH and AFC discordancy in the four age categorieswere $12.72 \%(36 / 283)$, $17.79 \%$ (87/489), 23.83\% (51/214), and 21.48\% (29/135), respectively. There were statistically significant differences. Figure 1 showed that oocyte yield was highest in Group A and lowest in Group D among all age categories. Although there were no statistically significant differences in oocyte yield between Group B and Group C, oocyte yield was higher in Group B than in Group C in all age categories except for the $\leq 30.0$ age category. In all age groups, the clinical pregnancy rate of Group B was higher than that of Group $C$, and there were significant differences in the 38.0-41.9 age categories.

Table 1 Comparison of clinical data of patients in different groups

\begin{tabular}{|c|c|c|c|c|c|}
\hline Parameters & $\begin{array}{l}\text { Group A } \\
(n=611)\end{array}$ & $\begin{array}{l}\text { Group B } \\
(n=85)\end{array}$ & $\begin{array}{l}\text { Group C } \\
(n=118)\end{array}$ & $\begin{array}{l}\text { Group D } \\
(n=307)\end{array}$ & $p$ value \\
\hline Age (years) & $32.69 \pm 4.59$ & $33.98 \pm 4.89 \boldsymbol{\Delta}$ & $36.81 \pm 4.79 \boldsymbol{\Delta}$ & $37.74 \pm 5.16$ & $<0.001$ \\
\hline Basal FSH (mIU/mL) & $7.48 \pm 2.20$ & $8.81 \pm 2.87 \boldsymbol{\Delta}$ & $10.14 \pm 4.57 \boldsymbol{\Delta}$ & $11.24 \pm 4.83$ & $<0.001$ \\
\hline No. of AFCs (n) & $12.29 \pm 4.08$ & $8.47 \pm 1.72 \boldsymbol{\Delta}$ & $4.53 \pm 1.41 \boldsymbol{\Delta}$ & $3.69 \pm 1.59$ & $<0.001$ \\
\hline AMH level (ng/ml) & $3.46 \pm 1.88$ & $0.76 \pm 0.24 \boldsymbol{\Lambda}$ & $1.94 \pm 1.03 \boldsymbol{\Delta}$ & $0.51 \pm 0.30$ & $<0.001$ \\
\hline Oocyte yield (n) & $10.63 \pm 5.14$ & $5.13 \pm 2.98 \boldsymbol{\Delta}$ & $4.11 \pm 2.50 \boldsymbol{\Delta}$ & $2.10 \pm 1.78$ & $<0.001$ \\
\hline Poor ovarian response (\%) & $5.07 \%(31 / 611)$ & $25.88 \% \boldsymbol{\Delta}(22 / 85)$ & $40.68 \% \mathbf{\triangle}(48 / 118)$ & $83.06 \%(255 / 307)$ & $<0.001$ \\
\hline Fertilization rate $(\%)$ & $72.41 \%(4702 / 6494)$ & $73.85 \%(322 / 436)$ & $73.20 \%(355 / 485)$ & $73.37 \%(474 / 646)$ & 0.862 \\
\hline Good-quality embryo rate (\%) & $41.01 \%(1961 / 4782)$ & $\begin{array}{c}45.51 \% \Delta \\
(152 / 334)\end{array}$ & $\begin{array}{c}35.28 \% \Delta \\
(145 / 411)\end{array}$ & $25.21 \%(121 / 480)$ & 0.004 \\
\hline Transplant cancellation rate (\%) $\star$ & $33.88 \%(207 / 611)$ & $32.94 \%(28 / 85)$ & $46.61 \%(55 / 118)$ & $68.73 \%(211 / 307)$ & $<0.001$ \\
\hline Clinical pregnancy rate (\%) & $\begin{array}{l}43.32 \% \\
(175 / 404)\end{array}$ & $43.86 \% \triangle(25 / 57)$ & $23.81 \% \triangle(15 / 63)$ & $25.00 \%(24 / 96)$ & $<0.001$ \\
\hline
\end{tabular}

$\Delta$ Group B and Group $C$ have significant differences. $(P<0.05)$

$\star$ The main reasons for transplant cancellation in the four groups were different

Group A: to prevent the occurrence of OHSS $(55.56 \%, 115 / 207)$

Group B: no transplantable embryos $(32.14 \%, 9 / 28)$ and to accumulate embryos $(32.14 \%, 9 / 28)$

Group C: no transplantable embryos $(29.09 \%, 16 / 55)$ and to accumulate embryos $(23.64 \%, 13 / 55)$

Group D: no transplantable embryos $(52.61 \%, 111 / 211)$ and to accumulate embryos $(28.44 \%, 60 / 211)$ 
Table 2 Clinical data of patients in different age categories

\begin{tabular}{|c|c|c|c|c|c|c|}
\hline age & Group & $\mathrm{N}$ & $\operatorname{AFC}(n)$ & $\mathrm{AMH}(\mathrm{ng} / \mathrm{ml})$ & Oocyte yield (n) & Clinical pregnancy rate (\%) \\
\hline$\leq 30.0$ & $A$ & 217 & $13.39 \pm 4.15$ & $3.83 \pm 2.06$ & $12.02 \pm 5.51$ & $44.68 \%(63 / 141)$ \\
\hline \multirow[t]{3}{*}{$(n=283)$} & B & 21 & $8.52 \pm 1.81$ & $0.78 \pm 0.27$ & $4.81 \pm 3.87$ & $33.33 \%(4 / 12)$ \\
\hline & C & 15 & $5.27 \pm 1.28$ & $2.30 \pm 1.31$ & $5.67 \pm 2.16$ & $22.22 \%(2 / 9)$ \\
\hline & $\mathrm{D}$ & 30 & $3.97 \pm 1.73$ & $0.54 \pm 0.24$ & $2.43 \pm 1.46$ & $26.67 \%(4 / 15)$ \\
\hline $30.1-37.9$ & A & 293 & $12.11 \pm 3.99$ & $3.39 \pm 1.81$ & $10.58 \pm 4.67$ & $45.78 \%(92 / 201)$ \\
\hline \multirow[t]{3}{*}{$(n=489)$} & B & 44 & $8.50 \pm 1.80$ & $0.73 \pm 0.25$ & $5.41 \pm 2.77$ & $47.06 \%(16 / 34)$ \\
\hline & C & 43 & $4.47 \pm 1.50$ & $1.89 \pm 0.62$ & $4.49 \pm 2.53$ & $36 \%(9 / 25)$ \\
\hline & $\mathrm{D}$ & 109 & $3.88 \pm 1.40$ & $0.58 \pm 0.30$ & $2.32 \pm 1.84$ & $32.5 \%(13 / 40)$ \\
\hline $38.0-41.9$ & A & 83 & $10.16 \pm 3.37$ & $2.89 \pm 1.52$ & $7.39 \pm 4.02$ & $31.48 \%(17 / 54)$ \\
\hline \multirow[t]{3}{*}{$(n=214)$} & B & 11 & $8.18 \pm 1.47$ & $0.77 \pm 0.19$ & $5.36 \pm 2.54$ & $66.67 \%(4 / 6)$ \\
\hline & C & 40 & $4.43 \pm 1.36$ & $2.12 \pm 1.34$ & $3.85 \pm 2.29$ & $15.00 \%(3 / 20)$ \\
\hline & $\mathrm{D}$ & 80 & $3.80 \pm 1.62$ & $0.49 \pm 0.31$ & $2.21 \pm 2.11$ & $27.27 \%(6 / 22)$ \\
\hline$\geq 42.0$ & A & 18 & $11.78 \pm 3.58$ & $2.53 \pm 0.88$ & $9.67 \pm 5.79$ & $37.50 \%(3 / 8)$ \\
\hline \multirow[t]{3}{*}{$(n=135)$} & B & 9 & $8.56 \pm 1.67$ & $0.86 \pm 0.11$ & $4.22 \pm 2.17$ & $20.00 \%(1 / 5)$ \\
\hline & C & 20 & $4.35 \pm 1.31$ & $1.43 \pm 0.51$ & $2.65 \pm 2.37$ & $11.11 \%(1 / 9)$ \\
\hline & D & 88 & $3.27 \pm 1.69$ & $0.43 \pm 0.29$ & $1.63 \pm 1.36$ & $5.26 \%(1 / 19)$ \\
\hline
\end{tabular}

\section{Discussion}

There are many indicators for predicting ovarian response. In general, with advancing age, FSH levels increase, and $\mathrm{AMH}$ levels and AFC decrease. In the course of clinical diagnosis and treatment, there are often discordances in relevant indicators. Previous studies have examined the concordance of FSH and AMH levels. Gleicher et al. investigated discordances between FSH and AMH levels in366 consecutive infertility patients. They found that oocyte yield declined in the following order: normal AMH and FSH levels, normal AMH but abnormal FSH levels, abnormal AMH but normal FSH levels, and abnormal AMH and FSH levels.
Therefore, they demonstrated that a normal AMH level is more important than a normal FSH level [10]. Leader et al. measured the AMH and FSH levels of 5354 infertile women from days 2 to 4 of the menstrual cycle and found that one in five women had discordant $\mathrm{AMH}$ and FSH values [11]. The AMH level and AFC are the two most accurate indicators for predicting ovarian response [7]. Discordances between them have not been reported in the literature.

In general, serum AMH levels are strongly positively correlated with AFCs [12]. Li et al. retrospectively studied 1156 women undergoing their first IVF cycle, and the results demonstrated that AMH level was significantly

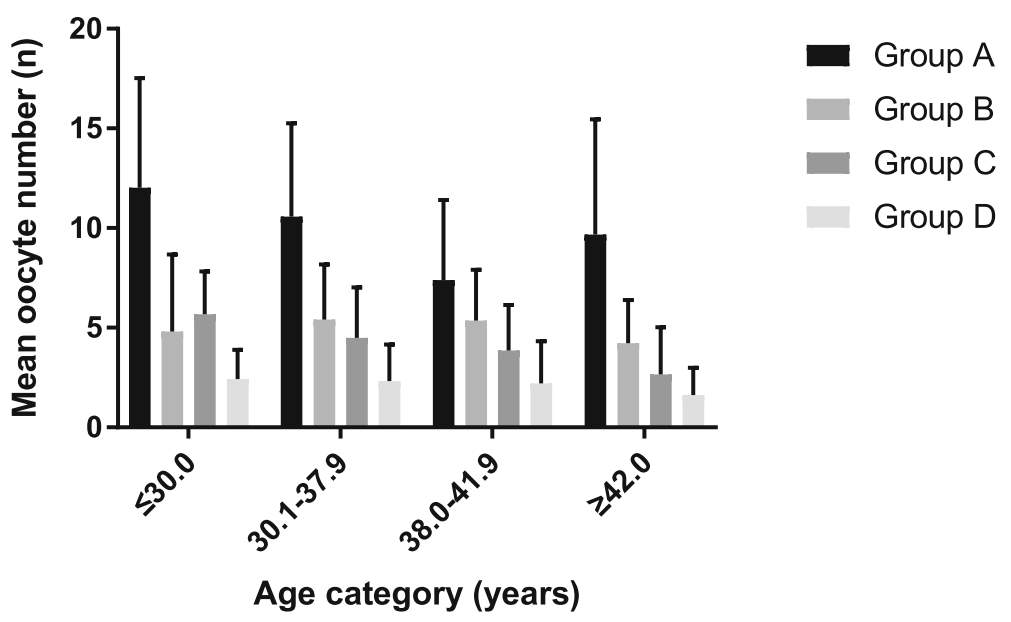

Fig. 1 Mean oocyte number in all age categories. Among all age categories, oocyte yield was highest in Group A and lowest in Group D. Although there were no statistically significant differences in oocyte yield between Group B and Group C, oocyte yield was higher in Group B than in Group C in all age categories except for the $\leq 30.0$ age category 
correlated with AFC. Both AMH and AFC showed significant correlations with age and ovarian response [13]. However, there are often discordances between the AMH level and AFC in clinical practice. In cases of discordance, which indicator should doctors choose to predict ovarian response and subsequently develop an ovulation induction protocol? Our results demonstrated that approximately one in five infertile women had discordance in the AFC and $\mathrm{AMH}$ level. The oocyte yield and clinical pregnancy rate were significantly higher in Group B (normal AFCs and low AMH levels) than in Group C (low AFC and normal AMH). The incidence of POR in Group B was significantly lower than that in Group C. According to the stratified analysis of age, for the three categories above the age of 30, oocyte yield was higher in Group B than in Group C. In all age categories, the clinical pregnancy rate of Group B was higher than that of Group C. The results suggest that a normal AFC is more important than a normal AMH value in predicting ovarian response. In other words, once the AFC becomes abnormal, even if the $\mathrm{AMH}$ level is still normal (Group C), the oocyte yield decreases. Therefore, we suggest that the AFC should be prioritized in the predicting of ovarian response and the clinical pregnancy rate in cases of discordance between the AFC and AMH level.

Why is there discordance between the AFC and AMH level? We speculate that this may be due to differences in the follicle population represented by the two indicators. The AFC refers to the number of follicles with diameters of $2 \mathrm{~mm}$ to $9 \mathrm{~mm}$. These follicles are mostly gonadotropin responsive and selectable for further growth and development through to the preovulatory stage during $\mathrm{COH}$. Therefore, the AFC is a direct marker of the recruitable follicular cohort [7]. AMH is a dimeric glycoprotein of the transforming growth factorb (TGF-b) family. AMH is produced by granulosa cells of pre-antral and small antral follicles of less than $4 \mathrm{~mm}$ diameter in the ovary [14]. AMH indirectly reflects the population of early growing follicles [15]. These small follicles are not able to enter the follicular recruitment stage during the ovulation induction cycle, let alone develop into mature follicles for egg retrieval and fertilization. Therefore, the AMH level mainly reflects the ovarian reserve function [16].

The female fertility decreases with increasing chronological age has been known for a long time. This agerelated decline is most likely due to a gradual decline in both oocyte quantity and quality $[17,18]$. According to the stratified analysis of age, in the category above the age of 42, oocyte yield in Group A (AFCs and AMH levels in the normal range) and Group B (normal AFCs and low AMH levels) showed no significant reduction compared with that in the other age groups, and clinical pregnancy rates reached 37.50 and $20.00 \%$, respectively.
Therefore, we suggest that for older infertile women, as long as the AFC is normal, IVF treatment should be actively performed, even if the AMH level is low.

\section{Conclusions}

In conclusion, the AFC and AMH level are the two most accurate indicators for predicting ovarian response and pregnancy outcomes. Our study demonstrated that approximately one in five patients in clinical practice had discordance in their AFCs and AMH levels. In view of the AFC being better than the AMH level for predicting POR, the AFC should be preferred in the prediction of ovarian response, to ultimately develop an optimal individualized $\mathrm{COH}$ protocol.

\section{Abbreviations}

AFC: Antral follicle count; AMH: Anti-Müllerian hormone; $\mathrm{COH}$ : Controlled ovarian hyperstimulation; $\mathrm{E}_{2}$ : Oestrogen; ELISA: Enzyme-linked

immunosorbent assay; FSH: Follicle stimulating hormone; HCG: Human chorionic gonadotropin; ICSI: Intracytoplasmic sperm injection; IVF: In vitro fertilization; OHSS: Ovarian hyperstimulation syndrome; PCOS: Polycystic ovarian syndrome; POR: Poor ovarian response; SD: Standard deviation; SPSS: Software Package for Social Sciences

\section{Acknowledgements}

We thank American Journal Experts (AJE) for English language editing. This manuscript was edited for English language by AJE.

\section{Authors' contributions}

$Y Z$ and $Y X$ conceived and coordinated the study, designed and analysed the experiments, and wrote the paper. QX, JS, XY and XS carried out the data collection and data analysis and revised the paper. YK, SW and CZ performed the experiments. All authors read and approved the final manuscript.

\section{Funding}

Not applicable.

\section{Availability of data and materials}

The datasets used and/or analysed during the current study are available from the corresponding author on reasonable request.

\section{Ethics approval and consent to participate}

This study was approved by the Clinical Research Institutional Review Board of Peking University First Hospital, and all patients provided informed consent.

\section{Consent for publication}

Not applicable.

\section{Competing interests}

The authors declare that they have no competing interests.

Received: 30 March 2019 Accepted: 28 June 2019

Published online: 04 July 2019

\section{References}

1. Nelson SM. Biomarkers of ovarian response: current and future applications. Fertil Steril. 2013;99:963-9.

2. Broer SL, Dolleman M, Opmeer BC, Fauser BC, Mol BW, Broekmans FJ. AMH and AFC as predictors of excessive response in controlled ovarian hyperstimulation: a meta-analysis. Hum Reprod Update. 2011;17:46-54.

3. Vural B, Cakiroglu Y, Vural F, Filiz S. Hormonal and functional biomarkers in ovarian response. Arch Gynecol Obstet. 2014;289:1355-61.

4. Broer SL, Mol BW, Hendriks D, Broekmans FJ. The role of anti-müllerian hormone in prediction of outcome after IVF: comparison with the antral follicle count. Fertil Steril. 2009;91:705-14. 
5. Cook CL, Siow Y, Taylor S, Fallat ME. Serum müllerian -inhibiting substance levels during normal menstrual cycles. Fertil Steril. 2000;73:859-61.

6. Kelsey TW, Wright P, Nelson SM, Anderson RA, Wallace WH. A validated model of serum anti-müllerian hormone from conception to menopause. PLoS One. 2011;6:e22024.

7. Jayaprakasan K, Campbell B, Hopkisson J, Johnson I, Raine-Fenning N. A prospective, comparative analysis of anti- Müllerian hormone, inhibin-B, and three-dimensional ultrasound determinants of ovarian reserve in the prediction of poor response to controlled ovarian stimulation. Fertil Steril. 2010;93:855-64.

8. Ferraretti AP, La Marca A, Fauser BC, Tarlatzis B, Nargund G, Gianaroli L. ESHRE consensus on the definition of 'poor response' to ovarian stimulation for in vitro fertilization: the Bologna criteria. Hum Reprod. 2011;26:1616-24.

9. Satwik R, Kochhar M, Gupta SM, Majumdar A. Anti- Müllerian hormone cutoff values for predicting poor ovarian response to exogenous ovarian stimulation in in-vitro fertilization. J Hum Reprod Sci. 2012;5:206-12.

10. Gleicher N, Weghofer A, Barad DH. Discordances between follicle stimulating hormone (FSH) and anti- Müllerian hormone (AMH) in female infertility. Reprod Biol Endocrinol. 2010;8:64.

11. Leader B, Hegde A, Baca Q, Stone K, Lannon B, Seifer DB, et al. High frequency of discordance between anti-müllerian hormone and folliclestimulating hormone levels in serum from estradiol-confirmed days 2 to 4 of the menstrual cycle from 5,354 women in U.S. fertility centers. Fertil Steril. 2012;98:1037-42.

12. Barbakadze L, Kristesashvili J, Khonelidze N, Tsagareishvili G. The correlations of anti-müllerian hormone, follicle-stimulating hormone and antral follicle count in different age groups of infertile women. Int J Fertil Steril. 2015;8:393-8.

13. Li HW, Lee VC, Lau EY, Yeung WS, Ho PC, Ng EH. Role of baseline antral follicle count and anti-Müllerian hormone in prediction of cumulative live birth in the first in vitro fertilisation cycle: a retrospective cohort analysis. PLoS One. 2013;8:e61095.

14. Chen Y, Ye B, Yang X, Zheng J, Lin J, Zhao J. Predicting the outcome of different protocols of in vitro fertilization with anti-Müllerian hormone levels in patients with polycystic ovary syndrome. J Int Med Res. 2017:45:1138-47.

15. Broekmans FJ, Knauff EA, Te VE, Macklon NS, Fauser BC. Female reproductive ageing: current knowledge and future trends. Trends Endocrinol Metab. 2007;18:58-65

16. Yarde F, Voorhuis M, Dolleman M, Knauff EA, Eijkemans MJ, Broekmans FJ. Anti-müllerian hormone as predictor of reproductive outcome in subfertile women with elevated basal follicle-stimulating hormone levels: a follow-up study. Fertil Steril. 2013;100:831-8.

17. Mutlu MF, Erdem M, Erdem A, Yildiz S, Mutlu I, Arisoy O, et al. Antral follicle count determines poor ovarian response better than anti-Müllerian hormone but age is the only predictor for live birth in in vitro fertilization cycles. J Assist Reprod Genet. 2013;30:657-65.

18. Te VE, Pearson PL. The variability of female reproductive ageing. Hum Reprod Update. 2002;8:141-54

\section{Publisher's Note}

Springer Nature remains neutral with regard to jurisdictional claims in published maps and institutional affiliations.

Ready to submit your research? Choose BMC and benefit from:

- fast, convenient online submission

- thorough peer review by experienced researchers in your field

- rapid publication on acceptance

- support for research data, including large and complex data types

- gold Open Access which fosters wider collaboration and increased citations

- maximum visibility for your research: over $100 \mathrm{M}$ website views per year

At $\mathrm{BMC}$, research is always in progress.

Learn more biomedcentral.com/submissions 